\title{
EFFECT OF ALCOHOL TO ORAL HEALTH
}

\author{
K. Peycheva and E. Boteva \\ Conservative Dentistry, FDM, MU - Sofia
}

\begin{abstract}
Summary. According to the World Health Organization there are almost two billion people worldwide who consume alcohol on a regular basis. It's a common abuse and almost 80 million are diagnozed with "alcohol abuse disorders" (WHO 2002, 2004). Excessive alcohol consumption is related to more than 60 different medical conditions, as suicide, homicide and different forms of accidents. Some conditions are acute, while other conditions such as liver cirrhosis, chronic pancreatitis, haemorrhagic stroke and various forms of cancer, are chronic consequences. Non-carious destructions of teeth like dental erosion are also associated with frequent alcohol consumption, because of precipitation of salivary proline-rich proteins caused by polyphenols present in most alcoholic drinks. The high concentration of organic and inorganic acids and the habit of keeping the alcoholic drink in the mouth can cause chronic inflammations of the soft tissues in the mouth and can increase the negative side effects from metals of crowns, bridges, orthodontic devises and various restorations. A literature review has been made due to the authors clinical observations and experiences.
\end{abstract}

Key words: alcohol, alcoholism, oral diseases, public health

\section{INTRODUCTION}

Whe term "alcohol" as used in medicine typically is applied to ethanol, rather than other alcohols. Ethanol $(\mathrm{CH} 3 \mathrm{CH} 2 \mathrm{OH})$ is the active ingredient in alcoholic beverages. For consumption purposes it is produced by fermentation of carbohydrates, such as simple carbohydrates in fruit and starch (which is previously hydrolysed during a process called malting) in grains, by yeasts. Spirits, such as whisky, brandy and vodka are produced by distillation of fermented products. Carbohydrate fermentation is incomplete in beer and complete in wine, with resulting alcohol contents between $3-8 \%$ and $7-18 \%$ volume $\%$, respectively. Distilled products, such as liqueurs and spirits, are $30 \%$ or greater alcohol volume $\%$ [4]. 
All alcohols are toxic, but ethanol is less toxic because humans body can metabolize it rapidly, which is related to the race, to general diseases, sex, physical activity, profession etc.. The various alcoholic drinks also contain substances that can act with or against ethanol. The limit between moderate and heavy alcohol drinking has been generally set at $40 \mathrm{~g} /$ day for spirits and is defined as tolerable upper level of alcohol intake (TUAL) [6]. Nevertheless, national drinking guidelines varis geographically, ethnically and is also related to culture, and education. In the UK the recommended alcohol consumption level is $\leq 2-3 \mathrm{AU} / \mathrm{day}$ ( $\mathrm{AU}$ alcohol units) for women (the number of $A U$ in various alcoholic drinks is displayed in Table 17.1), $\leq$ 3- $4 \mathrm{AU} /$ day for men and $\leq 1-2 \mathrm{AU} /$ day for pregnant women [11].

Most ethanol, rapidly absorbed through the gastric and duodenal mucosa, is metabolized by the liver, with a small fraction metabolized by oral and other mucosae of the upper digestive tract [20]. The enzyme acetaldehyde dehydrogenase $(A D H)$ catalyses ethanol oxidation to cetaldehyde, which is oxidized into non-toxic acetate by the enzyme alcohol dehydrogenase (ALDH). In the turnover, acetate is oxidized into fatty acids, carbon dioxide and water. Ethanol increases membrane permeability of the oral mucosa epithelial cells, thus promoting the penetration of other tobacco carcinogens such as nitrosonornicotine [23]. Alcohol drinking is also strongly associated with the risk of development of liver cirrhosis, which may result in impaired metabolism of carcinogens and in impaired immunity. A diet poor in fruits and vegetables is also typical for heavy drinkers [47].

\section{SYSTEMIC EFFECTS OF ALCOHOL, INFLUENCING OROFACIAL HEALTH: CARIES AND PERIODONTAL DISEASES}

Chronic alcoholics may neglect themselves as they are preoccupied with their addiction and often they may have poor dental hygiene. Alcoholics have a high incidence of decayed missing and filled teeth (DMF) and also more missing teeth compared to non-alcoholics [45]. Alcoholics have an increased rate of chronic, advanced generalized periodontal inflammations, gingivitis, bleading of the interdental papillae and deep gingival pockets with related bone losses. Addicts have worse dental hygiene indexes and dental index (based on both the number and severity of decayed, missing and filled teeth; 13.13 versus 4.74$)[3,35]$ Significantly fewer teeth and more active caries lesions are found in alcoholics and there is a trend for alcoholics to have more endodontically treated teeth than non drinking controls. No one has found so far significant differences in the number of periapical lesions in endodontically treated teeth. Horizontal bone loss and the presence of calculus is more frequent in alcoholic men than in alcoholic women [20,21]. Significantly more horizontal bone loss was observed in alcoholic non-smokers than in non-alcoholic non-smokers. In the non-smoking groups, alcoholics had significantly more periodontal destruction than non-smoking controls [15]. Age, low income, low education level, smoking and alcohol abuse seemed to be risk markers for periodontal destruction [30, 49, 50]. In another study, measures of oral hygiene, dental care and periodontal status were 
significantly worse and the number of teeth requiring treatment was higher in alcoholics with or without cirrhosis than in healthy subjects and in nonalcoholic patients with cirrhosis. Alcoholics had fewer teeth and more caries lesions than patients without alcohol abuse and healthy controls. The dental and periodontal status of patients with non-alcoholic cirrhosis did not differ from the control group [2].

\section{DIRECT EFFECTS OF ALCOHOL INFLUENCING OROFACIAL HEALTH: ENAMEL EROSION AND DRY MOUTH SYNDROME}

Several intrinsic factors are involved in the development of dental erosion, such as salivary flow rate, buffering capacity and salivary composition, pellicle formation, tooth composition and extrinsic factors, such as chemical $(\mathrm{pH}$, titratable acidity, phosphate and calcium concentration, fluoride content of the material in contact with the tooth) and behavioural factors (eating and drinking habits, lifestyle, excessive consumption of acids) [44,50]. Although low pH plays a crucial role in determining tooth wear and the evidence that acidic foodstuffs and beverages play a crucial role in the development of erosion is convincing, the density of acids in the food/ beverage. An important factor is the contact time between the acidic food/beverage and the dental surfaces [25, 49]. The $\mathrm{pH}$ of most alcoholic beverages is acidic, with values around $\mathrm{pH} 4,0$ and the concentration of organic and inorganic acids is high. It is important that the $\mathrm{pH}$ of incompletely fermented drinks, such as beer and wine, is acidic because it helps prevent contamination by other microorganisms, while the concentration of carbonic acid in alcopops (and in cocktails) is high because of the presence of soft drinks or fruit juices in the beverage [20]. Most alcoholic beverages are involved in determining dental erosion, but the major role is for alcopops [32, 37, $38,39]$ and cocktails $[10,34]$, which combine the erosive potentials of primary alcoholic beverages and of soft drinks and/or fruit juices. Among primary drinks, white wines are more erosive than red wines for the teeth, because of their higher concentration of titratable acids (36), while beers [22, 31] and ciders [36] have a moderate erosive potential. The astringency of alcoholic beverages is likely to be another factor promoting tooth wear. The taste typical of some alcoholic beverages is due to presence of high levels of polyphenols, mostly tannins, which binds salivary proteins, such as proteins and mucopolysaccharides, causing their precipitation, with consequent sensation of astringency, losses of lubrication of the oral mucosa and teeth and simultaneous decreasing protection of teeth from acids [12].

Discomfort of the teeth is acknowledged to be a problem for those people making or tasting wines regularly, due to wine's acidity [8, 9, 16, 17, 27, 28, 29, 46]. In fact, dental erosions are most frequent among wine merchants [10], winetasters [48] and winemakers in general [9], they keep wine in their mouth for longer time, as to be considered an occupational hazard. The erosive effects vary between wines [8]. Riesling style wine is more erosive than champagne style and both are more than claret [29]. Most white wines tested were at least as erosive as orange juice, while some wines were significantly more erosive than orange juice. Red wines may also 
be erosive $[7,24]$ and all ciders tested are acidic and had considerable erosive potential in vitro which was comparable to the one of orange juice [36]. Many commercially available drinks also have considerable erosive potential [35] as do alcoholic soft drinks [32] such as Hooch alcoholic lemonade [37]. Fluoride gels or varnishes may significantly reduce such enamel erosions [19]. Dental erosion is strongly associated with drinking behaviour. In fact, it is more frequent and severe among those whose alcohol consumption is continuous rather than in the form of episodic drinks $[10,33,38]$ and it is affected by the time the drink is kept into mouth before swallowing. Several epidemiological studies report a time-dependent association between chronic alcoholism and dental erosion, independently of socioeconomic status, with prevalence values as high as $50 \%[3,18,42]$. The typical dental erosions of heavy alcohol drinkers generally affects the palatal surfaces of the upper anterior teeth [43]. Summarizing, the evidence of the association between alcoholic beverage intake and dental erosion is convincing, with secondary drinks showing greater erosive potential than primary drinks and with an important role of drinking behaviour, particularly those habits characterized by frequent intake and long retention of the drink in the oral cavity.

Dental erosion is related to duration of alcoholism irrespective of confounding control of dental health behaviour and social situation [18]. Tooth erosion may arise from gastritis and reflux [48]. The teeth in alcoholic patients have significantly more wear than age- and sex-matched controls. Tooth wear is most marked in males and in those whose alcohol consumption was continuous. Erosive lesions appeared in $40 \%$ of the sample and areas affecting the palatal surfaces of the upper anterior teeth [38].

\section{DRY MOUTH}

The effects of alcohol on salivation have not been clearly defined [5]. The sublingual gland showed a proportional increase in adiposity and reduction in fibrovascular tissues but no noticeable reduction in its proportional volume [39]. The salivary findings of increased flow rate, protein and amylase levels indicate that hypertrophy and increased acinar function may be a component of the parotid enlargement and fatty replacement of functional gland tissues is probably not involved [1, 13]. Reduction in parotid salivary flow rate was associated with a statistically significant decrease in total protein and amylase secretion in a cohort group of patients [14]).

\section{ABSTINENCE OR WITHDRAWAL SYNDROME}

If the supply of alcohol is reduced or cut off, or if blood alcohol levels fall rapidly, the abstinence or withdrawal syndrome (AWS) may appear. Common alcohol withdrawal symptoms are morning 'shakes', typically trembling of the hands and body. Morning nausea and vomiting are sometimes called 'toothbrush heaves'. Alcoholics frequently resort to a drink first thing in the morning to relieve these withdrawal 
symptoms ('the morning eye-opener' or 'morning livener'). Other alcohol withdrawal symptoms are: diarrhoea, sweating, rapid pulse and raised blood pressure, confusion - disorientation in time and place, agitation - from mild anxiety to terror, illusions, hallucinations $[40,41]$.

\section{IN CONCLUSION}

High alcohol consumption has a deep impact on oral health. Some systemic alcohol-related conditions may indirectly affect oral health [40, 41]. These are the cases of missing teeth caused by caries or periodontal disease among chronic alcoholics, who generally neglect themselves and have poor oral hygiene. Tooth erosion may be a result from gastric reflux; of stomatitis caused by several micronutrient deficiencies; of sialosis and dry mouth with their particular impacts on dental caries development. In summary: high alcohol consumption has a direct impact on oral health. The side effects of heavy drinking are also risks of developing cancer of the oral mucosa, which is also in higher proportions among alcoholics. The dangers may increase due to the use of alcohol-containing mouthwashes and mouthrinses.

The high concentration of organic and inorganic acids and the habit of keeping the alcoholic drink in the mouth can cause chronic inflammations of the soft tissues and can increase the negative side effects from metals of crowns, bridges, orthodontic devises and various metal restorations.

\section{REFERENCES}

1. Abelson D, I Mandel, M Karmiol, Salivary studies in alcoholic cirrhosis, Oral Surg Oral Med Oral Pathol, 1976, 41, 188-92.

2. Allen J, W Eckardt, J Wallen, Screening for alcoholism: techniques and issues, Public Health Rep, 1988, 103, 586-92

3. Araujo M, K Dermen, G Connors, S Ciancio, Oral and dental health among inpatients in treatment for alcohol use disorders: a pilot study, J Int Acad Periodontol,2004 6, 125-30.

4. Berger A, Why wine might be less harmful than beer and spirits, Br Med J, 1998,317, 848.

5. Berry Mr, J Scott, Functional and structural adaptation of the parotid gland to medium-term chronic ethanol exposure in the rat, Alcohol, 1990, 25, 523-31.

6. Burger M, A Bronstrup, K Pietrzik, Derivation of tolerable upper alcohol intake levels in Germany: a systematic review of risks and benefits of moderate alcohol consumption, Prev Med, 2004, 39, $111-27$

7. Chaudhry Si, JI Harris, Sj Challacombe, Dental erosion in a wine merchant: an occupational hazard?, Br Dent J, 1997,182, 226-8.

8. Chikte Um, S Grobler, Tj Kotze, 'In vitro human dental enamel erosion by three different wine samples, South Afr Dent J, 2003, 58, 360-2.

9. Chikte Um, S Naidoo, Tj Kolze, S Grobler, Patterns of tooth surface loss among winemakers'. South Afr Dent J, 2005,60, 370-4.

10. Chuajedong $P, U$ Kedjarune-leggat, $V$ Kertpon, $V$ Chongsuvivatwong, et al, Associated factors of tooth wear in southern Thailand, J Oral Rehabil,2002, 29, 997-1002. 
11. Department of health, Sensible Drinking: Report of an inter-departmentalworking group, 1995, DH, UK.

12. Dreosti I, Antioxidant polyphenols in tea, cocoa, and wine, Nutrition, 2000,16, 692-4.

13. Dutta Sk, M Dukehart, A Narang, $P$ Latham, Functional and structural changes in parotid glands of alcoholic cirrhotic patients, Gastroenterology,1989, 96, 510-8.

14. Dutta Sk, M Orestes, S Vengulekur, P Kwo, Ethanol and human saliva: effect of chronic alcoholism on fl ow rate, composition, and epidermal growth factor, Am J Gastroenterol,1992, 87, 350-4.

15. Enberg N, J Wolf, A Ainamo, H Alho, et al, Dental diseases and loss of teeth in a group of Finnish alcoholics: a radiological study, Acta Odontol Scand, 2001,59, 341-7.

16. Ferguson M, Dunbar Rj, Smith Ja, Wall Jg, 'Enamel erosion related to winemaking, Occup Med, 1996,46, 159-62.

17. Gray A, Mm Ferguson, Jg Wall, Wine tasting and dental erosion, Case report. Aust Dent J, 1998,43, 32-4.

18. Hede B, Determinants of oral health in a group of Danish alcoholics'. Eur J Oral Sci, 1996,104, 403-8.

19. Jones L, D Lekkas, D Hunt, J Micintyre, et al, 'Studies on dental erosion: An in vivo-in vitro model of endogenous dental erosion - its application to testing protection by fl uoride gel application, Aust Dent J, 2002,47, 304-8.

20. Klampfl Cw, W Buchbergera, Pr Haddad, Determination of organic acids in food samples by capillary zone electrophoresis, J Chromatogr A, 2000, 357-64.

21. Kranzler Hr, Tf Babor, L Goldstein, J Gold, Dental pathology and alcohol-related indicators in an outpatient clinic sample, Community Dent OralEpidemiol, 1990, 18, 204-7.

22. Lissera Rg, L Maldonado, Lj Battellino, In vitro erosive capacity of some fruit juices and soft or low alcoholic strength beverages on human teeth, Acta Odontol Latinoam, 1998,11, 55-71.

23. Llewellyn Cd, Nw Johnson, $\mathrm{K}$ Warnakulasuriya, Risk factors for squamous cell carcinoma of the oral cavity in young people - a comprehensive literature review, Oral Oncol, 2001, 37, 401-18.

24. Lupi-pegurier L, M Muller, E Leforestier, Mf Bertrand, et al, In vitro action of Bordeaux red wine on the microhardness of human dental enamel, Arch Oral Biol, 2003, 48,141-5.

25. Lussi A, T Jaeggi, D Zero, The role of diet in the aetiology of dental erosion, Caries Res, 2004, 38(Suppl. 1), 34-44.

26. Mandel L, Dental erosion due to wine consumption, JADA, 2005, 136, 71-5.

27. Mandel L, H Baurmash, Parotid enlargement due to alcoholism, JADA, 1971,82, 369-71.

28. Mandel L, D Hamele-bena, Alcoholic parotid sialadenosis, JADA, 1997, 128, 1411-5.

29. Mok Tb, J Micintyre, D Hunt, Dental erosion: in vitro model of wine assessor's erosion, Aust Dent J, 2001, 46, 263-8.

30. Niquille M, B Burnand, P Magnenat, F Paccaud, et al, Dental disease among alcoholic individuals: a comparative study of hospitalized patients, J Gen Intern Med, 1993, 8, 470-5.

31. Nogueira Fn Dn, Souza, J Nicolau, In vitro approach to evaluate potential harmful effects of beer on teeth, J Dent, 2000, 28, 271-6.

32. O'sullivan Ea, M Curzon, 'Dental erosion associated with the use of "alcopop" - a case report, $\mathrm{Br}$ Dent J, 1998, 184, 594-6.

33. Rafeek M, S Marchan, A Eder, 'Tooth surface loss in adult, Int J Dent, 2006, 56, 186-193

34. Rafeek M, S Marchan, A Eder, W Smith, Tooth surface loss in adult subjects attending a university dental clinic in Trinidad, Int J Dent, 2006, 56, 181-6.

35. Rees Js, Fj Davis, An in vitro assessment of the erosive potential of some designer drinks, Eur J Prosthodont Restor Dent, 2000, 8, 149-52.

36. Rees Js, T Griffi, An in vitro assessment of the erosive potential of conventional and white ciders, Eur J Prosthodont Restor Dent, 2002,10, 167-71. 
37. Rees Js, K Burford, T Loyn, The erosive potential of the alcoholic lemonade Hooch, Eur J Prosthodont Restor Dent, 1998, 6, 161-4.

38. Robb Nd, Bg Smith, Prevalence of pathological tooth wear in patients with chronic alcoholism, $\mathrm{Br}$ Dent J, 1990,169, 367-9.

39. Scott J, K Woods, P Baxter, Salivary flow rate, protein and electrolyte concentrations in chronic alcoholic patients, J Biol Buccale, 1988b, 16,215-8.

40. Smith Aj, Rj Hodgson, K Bridgeman, Jp Shepherd, A randomized controlled trial of a brief intervention after alcohol-related facial injury, Addiction, 2003, 98, 43-52.

41. Smith $\mathrm{Aj}$, Jp Shepherd, Rj Hodgson, Brief interventions for patients with alcohol-related trauma, $\mathrm{Br}$ J Oral Maxillofac Surg, 1998, 36, 408-15.

42. Smith B, N Robb, Dental erosion in patients with chronic alcoholism, J Dent, 1989, 17, 219-21.

43. Smith $\mathrm{Ng}$, Prevalence of pathological tooth wear in patients with chronic alcoholism, $\mathrm{Br}$ Dent $\mathrm{J}$, 1990,169, 367-9.

44. Sorvari R, J Meurman, P Alakuijala, R Frank, Effect of fluoride varnish and solution on enamel erosion in vitro, Caries Res, 1994, 28, 227-32.

45. Who, World Health Report 2002: reducing risks, promoting healthy life. WHO, Geneva.

46. Who, Department of Mental Health and Substance Abuse, Global StatusReport on Alcohol, 2004. WHO, Geneva.

47. Wiktorsson Am, M Zimmerman, B Angmar - Månsson, Erosive tooth wear: revalence and severity in Swedish winetasters, Eur J Oral Sci, 1997,105, 544-50.

48. Young Wg, Tooth wear: diet analysis and advice, Int Dent J, 2005, 55, 68-72.

49. Zero Dt, A Lussi, Erosion - chemical and biological factors of importance to the dental practitioner, Int Dent J, 2005, 55(4Suppl.1), 285-90.

50. Zero Dt, A Lussi, Behavioral factors,Monogr Oral Sci, 2006, 20, 100-5.

\author{
Corresponding author: \\ Kalina Peycheva \\ Department Conservativ Dentistry \\ Faculty of Dental Medicine \\ Medical University \\ 1 Sv. G. Sofiyski St. \\ $B G-1431$ Sofia
}

